\title{
A Comparison of Courtship Behaviors of Two Generations of HBCU Male Students in Georgia
}

\author{
Yolonda Morgan \\ College of Arts \& Sciences, Albany State University \\ 504 College Drive, Georgia 31705, United States \\ yolo9653@gmail.com \\ Pamela Pitman Brown (Corresponding author) \\ College of Arts \& Sciences, Albany State University \\ 504 College Drive, Georgia 31705, United States
}

Tel: 1-229-500-2165 E-mail: pamela.brown@asurams.edu

The research was funded through the Center for Undergraduate Research at Albany State University.

\begin{abstract}
This paper is the completion of a replication of an earlier project which contributes to research on families, specifically courtship behaviors, attitudes, and strategies by young men of color in dating and searching for a life partner. Prior research indicates that males select female partners on physical attractiveness, and sex role socialization. Data collection via SurveyMonkey obtained 68 respondents, and a final sample size of 56 after filtering via four screening measures. The adjusted sample size of 48 participants ensured that all of those in the sample identified as single, heterosexual, Black/African American males. Data obtained is compared to data from 1948 with a similar population. Preliminary results indicate that the top two reasons for dating are companionship and finding a suitable life partner/mate. Spearman's rho correlation indicated several statistically significant monotonic relationships between the "Appropriate Behavior in Courtship" statements. Limitations include validity/reliability of the measure of "courtship behaviors" statements as it was not created as a scale, and items were unable to be reverse coded due to a lack of additional information from the original study.
\end{abstract}

Keywords: courtship, dating, marriage, Historically Black Colleges \& Universities, African American Males

DOI: $10.7176 /$ RHSS/10-4-14

Publication date: February $29^{\text {th }} 2020$

\section{Introduction}

Courtship behaviors, particularly college dating and courtship behaviors, are dictated by cultural norms and values, including those within the university culture itself. New rules may apply relative to changes and shifts in technology, including cellular phones and immediate messaging systems. Using questions from Willie (1949), the project aims to assess if there are courtship behaviors, which have shifted or are similar, using updating to newer terminology and technological use.

The study contributes to the existing knowledge in the field of sociology and psychology based on prior work completed by Dr. Charles Vert Willie within his Master's thesis (1949) and the continuation of his work on African American marriages and families. The study assists in the research on families, focusing on the courtship behaviors that lead to coupling, by studying the modern day courtship behaviors and strategies of traditional college-aged men, specifically at an HBCU. Additionally it assist us in identifying shifts in courtship behaviors from Dr. Willie's original work or if the previous courtship behaviors are similar/alike but with different "named" components.

Participants were recruited from HBCU traditional-aged (18-25) heterosexual men who currently attend Albany State University. The students received a definition of "formal courtship" for the purposes of maintaining constant understanding of the term. For the current study we defined "formal courtship" for the purposes of the study as: $a$ relationship prior to engagement or marriage, but with a formal understanding between the two parties involved in matters concerning monogamy, shared expectations of duration of time before the formal engagement, and where the relationship is formally announced or understood as an arrangement with family/friends.

The definition was posed to the respondents prior to entering the survey. The internet survey based SurveyMonkey 
collected participants' answers. Participating male students provided information on how they learned about courtship/dating rituals, who taught them the guidelines within the culture, and what criteria must a woman have in order to consider forming a courting relationship.

Prior research indicates that male mate selection values physical attractiveness and youth (Fisman, Iyengar, Kamenica and Simons 2006; Russock 2011). Additional research (Shoemake 2007) indicates that men exhibit gender specific preferences for mate criteria based on sex role socialization. The study hopes to assess if males in the sample have traditional ideals in relationships or a more modern updated generational view of relationships. Recruitment techniques include contact via professors; flyers posted in common areas at ASU, emails, and Facebook or other social media recruitment methods.

The goals of the research are exploratory and knowledge based. Our research from an exploratory standpoint hopes to firstly, uncover what the ASU men believe are appropriate courtships behaviors and how or if these beliefs align with the Morehouse men of 1948. Secondly, our research is interested in who taught the ASU men about dating relationships with women, and future marriage. Thirdly, we hope to learn the three major reasons for the ASU men to participate in dating/courting, as well as the dating difficulties they experience. Next, we hope to find out more about their current relationship status and the intricacies of that relationship, if any. Finally, we hope to be able to identify if there are differences or similarities in the courtship and dating behaviors from the HBCU men of 1948 and the HBCU men of 2018.

\section{Literature Review}

The study by Williamson (1965) surveyed 530 students (295 males and 235 females) that attended a college or university in Philadelphia. The research study was meant to address the role of subcultures social class and religion, in determining attitudes on marriage. It was also meant to address if the concept of an "ideal mate" really exists and the definition of ideal mate. In Williamson's study the average age for most of the participants was 20.9 years and the marital status of the students was considered. The researchers assigned the students a social class based on their father's information (Williamson, 1965). Participants were organized by their classes; lower class, lower middle class, middle class, upper middle class, and upper class (Williamson, 1965). They were also organized according to their religions; Protestant, Catholics, Jews, and others who did not identify with any religion (Williamson, 1965).

The participants were asked the importance of different factors such as: lack of mutual interests, lack of selfconfidence, inability to communicate, insufficient time for dating, no opportunity to date, conversation too casual, dates lack attractiveness or status, and degree of turn- over in dates (Williamson, 1965). It was found that there were differences in responses based on the participants' marital status (Williamson, 1965). As far as class it was found that upper and middle class girls placed more emphasis on personality development being their motive for marriage (Williamson, 1965). It was also found that Jewish men placed emphasis on sexual opportunities during marriage more than any other male of any religion (Williamson, 1965). Jewish girls reported less premarital sex than any other religion (Williamson, 1965). Overall more emphasis was placed on the subculture of sex rather than class and religion (Williamson, 1965). Based on the findings of Williamson (1965), one might expect the participants of the current study to place more emphasis on willingness of sexual activity by one's partner.

Klaus, Hersen, and Bellack, (1977) surveyed 90 males and 105 females from the University of Pittsburgh with 20 being the average age of the students. The students participated in a research study, addressing their dating habits, interests, and attitudes, by responding to a 35-item questionnaire (Klaus, Hersen, \& Bellack, 1977). The survey asked for information on frequency of dating behavior, desirable dating patterns, and dating issues (Klaus et al., 1977). An additional question asked for the stage of the relationship sexual activities occurred, along with a working definition of dating (Klaus et al., 1977). The researchers were able to lessen or eliminate biases prior to survey distribution, by ensuring students confidentiality (Klaus et al., 1977).

Klaus et al. (1977) found that in all of the sections there were considerable differences between men and women in dating and relationships. Research indicated males stated sexual activities began on the third date, with females stating they weren't participating at all or not until the 20th date (Klaus et al., 1977). The research indicated that both sexes agreed on the earliest date to discuss family matters, sex, and marriage, and both agreed on the difficulty of dating (Klaus et al., 1977). Considering the findings of Klaus, Hersen, and Bellack, (1977), one might expect males of the current study opinions' on dating and relationships to differ from the women of that same HBCU.

The more recent study by Schwarz and Hassebrauck (2012) examined the mate selection preferences of 21,245 heterosexual men and women were not in closed relationships. The age range for the participants was 18 to 65 
years of age with 41 being the average age (Schwarz \& Hassebrauck, 2012). The researchers employed the use of an online questionnaire that contained 82 mate selection questions, the participants were asked to select from (Schwarz \& Hassebrauck, 2012). Within the questionnaire the participants were asked to select the importance of that specific criteria rating it on a scale of 1 to 5 , with 1 being unimportant and 5 being very important (Schwarz $\&$ Hassebrauck, 2012). The questions were used as a way to determine the criteria that would be needed for the participant to engage in a long-term relationship (Schwarz \& Hassebrauck, 2012). They then was asked the age range for which they would select their mate from (Schwarz \& Hassebrauck, 2012). Lastly they were asked to answer demographic information (Schwarz \& Hassebrauck, 2012). It was found that women required more from their partners and men placed more emphasis on physical attractiveness (Schwarz \& Hassebrauck, 2012). Taking in consideration the findings of Schwarz \& Hassebrauck (2012), one might expect the males of the current study to favor physical attractiveness over any other criteria.

Willie's (1949) thesis, A Study of Courtship Patterns of College Men, provides a research lens into male students at an HBCU in Georgia (Morehouse College), discovering their courtship patterns. One hundred-ten male students at Morehouse College in Atlanta, enrolled during the Spring 1948 semester were surveyed (Willie, 1949). Willie employed a mixed method approach to his discoveries, using interviews and survey data. Willie (1949) was able to determine the major reasons men named for participating in courting (Willie, 1949), companionship, finding a suitable mate and physical satisfaction. He also found that over half the males dated frequently from one to three times a week, and over two-thirds of these men dated women who were not located on college campuses (Willie, 1949).

\section{Methods}

\subsection{Design}

The sampling methodology is a non-probability purposive snowball sampling. We used the internet survey tool, SurveyMonkey, to collect the data and to obtain consent. A consultation with the university's Institutional Review Board (IRB) protocol on internet surveys (Albany State University IRB Approval \# 1221316-2) garnered approval in early Fall 2018 on the consent procedure involving the internet data collection. Thus, answering "Accept" to the statement "By clicking ACCEPT your consent to participate is implied," is consent of participation.

\subsection{Participants}

Male students who were eligible for participation were between the ages of 18-25 and current students of Albany State University. Thirteen hundred (1,300) students met the criteria of male, 18-25, and current students of ASU per the ASU Informational Technology Service. Additional inclusion criteria was a self-identification as a heterosexual. Exclusion criteria through filtering questions included if the respondent is female, not a current student of Albany State University, outside of the age parameters, and identification as a non-heterosexual. As the research is investigating male and female relationships, the exclusion of identification as a non-heterosexual is critical to the survey. Based on an a priori power calculation using $\mathrm{G}^{*}$ power assuming an alpha=.05 and a correlation: bivariate normal model, using $\mathrm{N}<1300$, a sample of 38 participants would be adequate (Erdfelder, Faul, and Buchner, 1996).

The initial entrance to the survey allowed for the recruits to be asked to "accept" or to "decline" entrance using the concept of implied consent, by clicking an "accept" button to enter the filter questions of the survey. If the recruit chose to "decline" entrance or thus not consent to participation, they were directed to an exit from the survey page.

A total of 68 respondents consented to enter the survey. Respondents who did not meet the inclusion criteria were filtered through a series of four screening measures (See Appendix A, Figure 1). If the respondent did not meet qualifications after the filter questions, they were redirected to a survey exit page thanking them for their assistance. This procedure has been used in several projects such as Brown and Ewen (2012), and Brown (2012). Additionally, Mustanski (2001) states "it is preferable to simply ask participants about all relevant characteristics and then filter out those who do not meet the study requirements" to prevent sabotage of the internet-based survey.

\subsection{Sample \& Recruitment}

\subsubsection{Internet/Social Media Recruitment}

Recruitment techniques employed were solicitations on ASU Facebook pages of the Sociology and Psychology departments, Psychology and Sociology course lists, and an email list of male undergraduates who were of traditional age provided via Internet Technology at ASU, as well as recruitment flyers. Recruitment using Facebook 
has been noted in other publications as just one way to gather survey data when there is a self-selection bias (Brown, 2012; Brown \& Ewen, 2012).

\subsection{Measures}

\subsubsection{Demographic Measures}

Within demographic measures, the categories were race, college rank, age, religion, and veteran status. For race, Students were asked to self-identify race, with the following choices: Black/African/African American (both Hispanic and non-Hispanic), Afro-Caribbean, White/European/Caucasian (both Hispanic and non-Hispanic), American Indian/Alaskan Native, Asian, and Native Hawaiian, or other Pacific Islander. Students were able to indicate any combination of the above. For College Rank, students chose from the four college ranks of freshman, sophomore, junior, and senior. Students were able to self-identify their college rank placement. For Age, students chose from the age of 18 to 25 in year increments. They were able to self-identify their age without use of a birth date. No birthdate data was collected, as with a small sample it might be possible to determine who an individual was as a participant. Religion offered options of Protestant, Catholic, Jewish, Muslim, Non-believer, and other. For Veteran Status, the question of "Are you a U.S. Veteran of the Armed Forces" was answered with a yes/no.

\subsection{Procedures}

\subsubsection{Data Collection}

SurveyMonkey offers a SSL encryption feature, whereby the researcher is able to permit, allowing the sensitive data to be protected as it moves along the communication pathway between the participants' computers and SurveyMonkey's servers. The participants' IP addresses are masked and are therefore not visible to the researcher by using SurveyMonkey's data protection protocol. During data collection, the data had protection via SurveyMonkey SSL encryptions. At the end of data collection and downloading the data, the downloaded data were stored in a password protected data file on a media storage device. A notification informed the IRB that the computer viewing the data had updated anti-virus protection, which is available to University faculty and students. After cleaning the data and the online materials, including the survey and responses, the site no longer held any data from the project. This was included as an added layer of protection for IP addresses.

\section{Results}

\subsection{Respondent Demographics}

The survey initially obtained $68(\mathrm{~N}=68)$ respondents. After eliminating respondents who did not meet the criteria via the four screening measures the final sample size was 56 . Data collection spanned a 70 day time frame during the late summer and early fall of 2018. Unfortunately, during the collection period, the campus experienced Hurricane Michael, and many students lost power, relocated off campus, lost access to the university email system, and endured additional hardships, as the University closed for a week for recovery.

With the overwhelming majority (85.7\%) of the final sample size stating they were African American, or biracial including African American, the authors further reduced the sample to include only those who included African American or Black as part of their self-identified racial identity. This lowered the sample to $48(\mathrm{n}=48)$ individuals which is adequate.

Demographic data Albany State University. Of the final lower sample of 48 participants, all were single, heterosexual males, who self-identified as Black/African American or mixed race including African American. Additionally, none of the 48 were veterans of the military. The majority of the participants were 19 years of age $(n=19,39.6 \%)$, and a majority were sophomores $(\mathrm{n}=17,35.4 \%)$. Additionally, an overwhelming majority $(\mathrm{n}=33$, $68.8 \%$ ) indicated that they were single and were currently not in a relationship. With regards to religious affiliation, a majority of the participants answered Christian/Protestant $(n=22,45.8 \%)$. Interestingly, nine participants $(18.8 \%)$ indicated they were non-believers, one (2.1\%) indicated Pagan, and eight (16.7\%) did not answer. See Table 1 for demographic data for Albany University male students.

Demographic data Morehouse students (Willie, 1949).

All of the participants within Willie's 1949 study were African-American and were single/unmarried $(n=110$, $100 \%)$. While none of the ASU students were veterans, Willie's study (1949, p. 12) indicated that $35.5 \%$ of the students were veterans, with the majority of the veterans being of junior class rank $(N=21)(n=11,52 \%)$. The majority of the participants were 19-20 years of age $(n=36,32.7 \%)$. With regards to religious affiliation, a majority of the participants answered Christian/Protestant $(n=95,86.4 \%)$, with the remainder indicating Catholic $(n=5$, $4.5 \%)$, Other $(n=4,3.7 \%)$ and no answer provided $(n=6,5.4 \%)$. See Table 2 for demographic data for Morehouse 
participants.

\subsection{Learning about dating, relationship with women and future marriage}

Positing a question of "who taught you about dating relationships with women, and future marriage", the majority of ASU students noted that their mother/stepmother $(n=28,58.3 \%)$ gave instruction. Students indicated father/stepfather $(n=24,50 \%)$ being second source of instruction. Interestingly, six students indicated only their mother/stepmother taught them with 17 indicating that both parents gave instruction. Table 3 indicates results of the survey.

\subsection{Reasons for dating}

The top two reasons for dating or courting for both ASU students and Morehouse students was the same, companionship $(70.8 \% ; 60.9 \%)$ and finding a suitable mate $(52.1 \% ; 31.8 \%)$. Within Willie's 1949 study, he indicated there were three main reasons for dating. Firstly, the Morehouse students noted companionship $(n=67$, $60.9 \%)$. The second two reasons were tied at 31.8\% $(n=35)$ finding a suitable mate and physical satisfaction. We constructed our survey with reasons for dating as a multiple answer question, which allows us to view all of the reasons and rank them in order as to the most common. Table 4 shows the percentages of both ASU and Morehouse students for "reasons for dating/courting."

\subsection{Dating Difficulties}

Willie (1949) noted that there were major difficulties in dating during the college years. For the Morehouse students in 1948, the two major difficulties, which interfered within their ability to date, were studying $(n=46$, $41.8 \%)$ and a lack of money $(n=37,33.6 \%)$. For the current ASU students, the two major difficulties were that they were too shy $(n=20,41.7 \%)$ and lacked social contact $(n=17,35.4 \%)$. In relation to the findings from Morehouse students, the ASU students ranked studying and a lack of money at third (tied; $n=6,12.5 \%$ ) in difficulties. Only $3(6.3 \%)$ ASU students indicated they played on a university sports team and had trouble dating due to those time constraints. Additional comments from ASU students concerning their dating difficulties included, never having dated, being a "bad texter", and conflicts due to "shared tastes, views, approach to relationships and interests". As far as those stating they had "no difficulties" dating, 23.6\% $(n=26)$ of the Morehouse students and $20.8 \%(n=10)$ of the ASU students answered in the affirmative.

\subsection{Initial Introduction to Women}

Willie (1949) noted there were three main ways that men met women, or an initial introduction. Within his study, the most frequent method for an introduction was via a mutual friend ( $n=59,53.7 \%)$, with self-introduction second ( $n=42,38.2 \%)$ and the women introducing themselves as the final $(n=4,3.6 \%)$. In the current study, the majority of the ASU participants indicated self-introduction as the most frequent method $(n=26,66.7 \%)$, followed by women introducing themselves $(n=3,7.7 \%)$; and mutual friend $(n=2,5.1 \%)$. This shows a generational shift in how men are meeting the women, as they are not relying on friendship connections for the majority of introductions. Additionally, we could add that the social norms indicating how men are to meet women, have shifted toward more self-initiation. However, it could also be that men are more likely to approach women with whom they have an initial attraction to for an initial conversation. Four men $(10.3 \%)$ in the ASU study indicated it was a combination of all three methods with two men indicating they met via social media (5.1\%).

\subsection{Site of first acquaintance}

Willie (1949) noted there were six sites where the men initially met the women they dated. They are in order dance $(n=54,49.1 \%)$, library $(n=34,30.9 \%)$, church $(n=33,30 \%)$, house party $(n=30,27.3 \%)$, friend's house $(n=$ $29,26.4 \%)$, and drug store $(n=16,14.5 \%)$. For the ASU participants, the initial available answers were different than those of Willie's (1949) study in that we knew social norms have shifted in where students now hang out. For example: drug store was eliminated, library was retained, church was retained, house party was retained (and no definition was given as to the term), friend's house was retained. We added a bar/nightclub and left out dances. School or school affiliated events was also added, as was social media. The top six for ASU participants was School or School affiliated events $(n=14,35.9 \%)$ with the second spot being other, as in not within the list $(n=$ $10,25.6 \%)$. House party was third $(n=6,15.4 \%)$, and friend's house/social media tied with three participants each $(n=3,7.7 \%)$. The final spot of sixth, only contained one participant per category in bar/nightclub, church, and the library $(n=1,2.6 \%)$. Clearly the focus on places for meeting has shifted over the past 70 years!

\subsection{Reconstruction of Dr. Willie's original eight statements of "Appropriate Behavior in Courtship"}

Using Dr. Willie's graduate thesis (1949), eight statements he asked of the Morehouse men were used in the survey. We reworded the statements for readability. For example, Dr. Willie used "One should never tell a girl all his 
business", which was rewritten as "You should never tell a woman all your business." We removed the use of "girl" and replaced the wording with "woman" within all of the statements. (Appendix B). A 3-point Likert scale from Disagree (1) to Agree (3) was used with the neutral measure (2) being asked as "neither agree nor disagree."

Within the "appropriate behavior in courtship" statements, 36 of the 48 participants responded. Table 5 shows how the ASU participants and the Willie's (1949) participants answer the statements. While Willie's (1949) work did not report the $n$ or frequency, but reported the percentage, a conversion calculation was performed $(\mathrm{Y}=\mathrm{P} \% * \mathrm{X})$ in order to convert the percentage to frequency, and then report both groups' valid percent.

\subsubsection{Mann-Whitney U Test Results of the eight statements of "Appropriate Behavior in Courtship}

\subsubsection{It does not pay to be nice to a woman}

Using a non-parametric independent samples test, Mann-Whitney U Test revealed significance between the groups of ASU and Morehouse students, as three statements within "Appropriate Behavior in Courtship," indicate reject the null hypothesis. Descriptive statistics showed that for the statement "It does not pay to be nice to a woman," that ASU participants $(m d=1.5$; mean rank=89.08) scored higher than Morehouse participants $(m d=1$; mean rank=66.25) indicating more neutrality in the statement. Mann-Whitney U-value was found to be statistically significant $\mathrm{U}=1311.00(\mathrm{Z}=-3.60), p<0.01$, and the difference between the ASU and Morehouse Groups was medium $(r=-.30)$.

\subsubsection{You should never think you are the only man dating the woman you are dating}

Descriptive statistics showed that for the statement "You should never think you are the only man dating the woman you are dating," that ASU participants $(\mathrm{md}=2$; mean rank=52.17) scored lower than Morehouse participants $(m d=3$; mean rank=78.67) indicating less agreement with the statement. Mann-Whitney U-value was found to be statistically significant $\mathrm{U}=1212.00(\mathrm{Z}=-3.87), p<0.01$, and the difference between the ASU and Morehouse Groups was medium $(r=-.32)$.

\subsubsection{You should never tell a woman all your business}

Descriptive statistics showed that for the statement "You should never tell a woman all your business," that ASU participants $(\mathrm{md}=2.5$; mean rank=48.47) scored lower than Morehouse participants $(\mathrm{md}=3$; mean rank $=79.92)$ indicating less agreement with the statement. Mann-Whitney U-value was found to be statistically significant $\mathrm{U}=1079.00(\mathrm{Z}=-5.96), p<0.01$, and the difference between the ASU and Morehouse Groups was large $(r=-.50)$.

\section{Discussion \& Conclusion}

The primary purpose of the current research was to identify the courtship and dating behavior of males at an HBCU. It also aimed to identify the shifts (if any) in courting behaviors. Previous studies suggested that males may favor physical attractiveness of their partner over anything else. It was also suggested the reason for courtship was for finding a suitable mate. In the current study, it was also found that males' reason for courtship was to find a suitable mate.

Our research from an exploratory standpoint hopes to firstly, uncover what the ASU men believe are appropriate courtships behaviors and how or if these beliefs align with the Morehouse men of 1948. Our findings indicate that the ASU/Morehouse men align in beliefs with the exception of one of the appropriate courtship items, whether men should assume all of the financial obligations of dating. The Morehouse men disagreed with the statement (over 50\%), but the ASU men were split across the agree/disagree/neutral answers with almost one-third in each category.

Secondly, our research is interested in who taught the ASU men about dating relationships with women, and future marriage. Parents/stepparents were acknowledged as the individuals who taught the ASU men about women, relationships, marriage, with mothers/stepmothers giving almost $60 \%$ of the participants' instruction.

Thirdly, we hope to learn the three major reasons for the ASU men to participate in dating/courting, as well as the dating difficulties they experience. Next, we hope to find out more about their current relationship status and the intricacies of that relationship, if any.

Finally, we hope to be able to identify if there are differences or similarities in the courtship and dating behaviors from the HBCU men of 1948 and the HBCU men of 2018. Williamson (1965) found that more emphasis was placed on the subculture of sex rather than class and religion. Based on the findings of Williamson (1965), it was expected that the participants of the current study would place more emphasis on willingness of sexual activity by one's partner. However the current study found that guys placed more emphasis on companionship and finding a 
suitable mate/life partner. The reason for the discrepancy might be due to the different cultural expectations. It may also be due to Williamson (1965) was conducted at a PWI while, the current study was conducted at an HBCU.

Klaus, Hersen, and Bellack, (1977) research indicated that both sexes agreed on the earliest date to discuss family matters, sex, and marriage, and both agreed on the difficulty of dating. Considering the findings of it was expected that males of the current study opinions' on dating and relationships would differ from the women of that same HBCU. Since the current study only surveyed males it would be difficult to determine if men and women would have different opinions on family matters, sex and marriage.

Schwarz and Hassebrauck (2012) research revealed that women required more from their partners and men placed more emphasis on physical attractiveness. Taking in consideration the findings of Schwarz \& Hassebrauck (2012), it was expected that the males of the current study would favor physical attractiveness over any other criteria. However, the males of the current study appeared to want companionship over anything else. The reason for the conflicting views may be Schwarz and Hassebrauck's (2012) study was conducted in Germany, while the current study was conducted in the United States.

Willie's (1949) thesis found that males had three main reason for dating; companionship, finding a suitable mate, and physical satisfaction. Considering the findings of Willie (1949), it was expected that males at an HBCU, main reasons for dating would also be companionship, finding a suitable mate, and physical satisfaction. In the current study, it was found that male's main reasons for dating was companionship and finding suitable mates as well. The studies were very similar almost parallel to one another. There were some differences in the questions that were asked.

While not a current goal of the project, we hope to be able to collect additional data at a later date from other University System of Georgia HBCUs and private HBCUs within Georgia to assess if the behaviors are similar or different across the state. As the project addresses an important and neglected area of research, the potential minimal risks appear to offset the potential knowledge gained from this work.

\subsection{Limitations}

An initial limitation realized is that the study has focused on HBCU males only, and is limited to Southwest Georgia. The university is situated within an urban setting of approximately 73,801 (U.S. Census, 2016) persons with $71 \%$ of the population being Black or African American. The area experiences a high level of poverty within its population. The population as noted in works by W.E.B. Dubois, as well as slavery prior to the Civil War historically molds the area. The University, which played a large part within the Civil Rights Movement (The Albany Movement, 1961-1962), headed by Dr. W. G. Anderson contributed much to the history as well. The Albany Movement was instrumental in leading Dr. Martin Luther King, Jr., Reverend Ralph Abernathy, and other civil rights leaders to Birmingham in 1962.

A second limitation is the lack of data acquired from Willie (1949) article. While the data were reconstructed via mathematical methodology, in no way were we able to truly have the data align with age or college rank in order to assess differences between the Morehouse data and the ASU data. Thus our observations are wholly resting on percentages.

An additional limitation is the validity or reliability of the measure of courtship behaviors. While would very much have liked to have used the data as a created scale, we were unable to do this. Furthermore, the questions were unable to be reverse coded due to the lack of knowledge of how Dr. Willie intended the coding other than Agree/Neutral/Disagree.

\section{References}

Bowman, H. A. (1960). Marriage for Moderns. New York: McGraw Hill Book Company.

Brown, P. P. (2012). Relationship Power and Control among Single Heterosexual Women at Midlife: Correlates of Condom Use and Traditional Gender Role Ideology. Electronic Dissertation. Retrieved from https://etd.ohiolink.edu/

Brown, P. P., and Ewen, H. (2012). "Utilization of Social Media to Recruit Adults Over 40 for Sensitive Research Topics." Presented at the Gerontological Society of America's (GSA) Annual Meeting (November 2012) in San Diego, California.

Cazenave, N. (1979). Middle-income black fathers: An analysis of the provider role. Family Coordinator, 28: 58393. 
Erdfelder, E., Faul, F., and Buchner, A. (1996). G-power: A general power analysis program. Behavior Research Methods, Instruments, \& Computers, 28: 1-11.

Fisman, R., Iyengar, S., Kamenica, E., and Simonson, I. (2006). Gender Difference in Mate Selection: Evidence from a Speed Dating Experiment. The Quarterly Journal of Economics. 121 (2):673-697.

Klaus, D., Hersen, M., and Bellack, A. S. (1977). Survey of dating habits of male and female college students: A necessary precursor to measurement and modification. Journal of Clinical Psychology, 33(2):369375.https://doi.org/10.1002/1097-4679 (197704)33:2<369:: AID-JCLP227 033 0209>3.0.CO;2-O

Muehlenhard, C. L., and McFall, R. M. (1981). Dating initiation from a woman's perspective, Behavior Therapy, $12,5,(682)$

Parks, M. R., Dindia, K., Adams, J., Berlin, E., and Larson, K. (1980). Communication apprehension and student dating patterns: A replication and extension, Communication Quarterly, 28, 2, (3).

Russock, H. (2011). An evolutionary interpretation of the effect of gender and sexual orientation on human mate selection preferences, as indicated by an analysis of personal advertisements. Behaviour, 148(3), 307-323. Doi: $10.1163 / 000579511 \times 556600$

Schwarz, S. and Hassebrauck, M. (2012). Sex and Age Differences in Mate Selection Preferences. Human Nature, 23(4): 447-466. doi:10.1007/s12110-012-9152-x

Shoemake, E. G. (2007). Human Mate Selection Theory: An Integrated Evolutionary and Social Approach. Journal of Scientific Psychology, 11, 35-41.

U. S. Census. (2016). Quick Facts Albany Georgia. Retrieved from https://www.census.gov/quickfacts /fact/table/albanycitygeorgia/INC110216 shoe

Williamson, R. (1965). Dating, Courtship and the 'Ideal Mate': Some Relevant Subcultural Variables. The Family Life Coordinator, 14(3): 137-143.

Willie, Charles Vert. (1949). A study of courtship patterns of college men. ETD Collection for AUC Robert W. Woodruff Library. Paper 928. 
Appendix A:

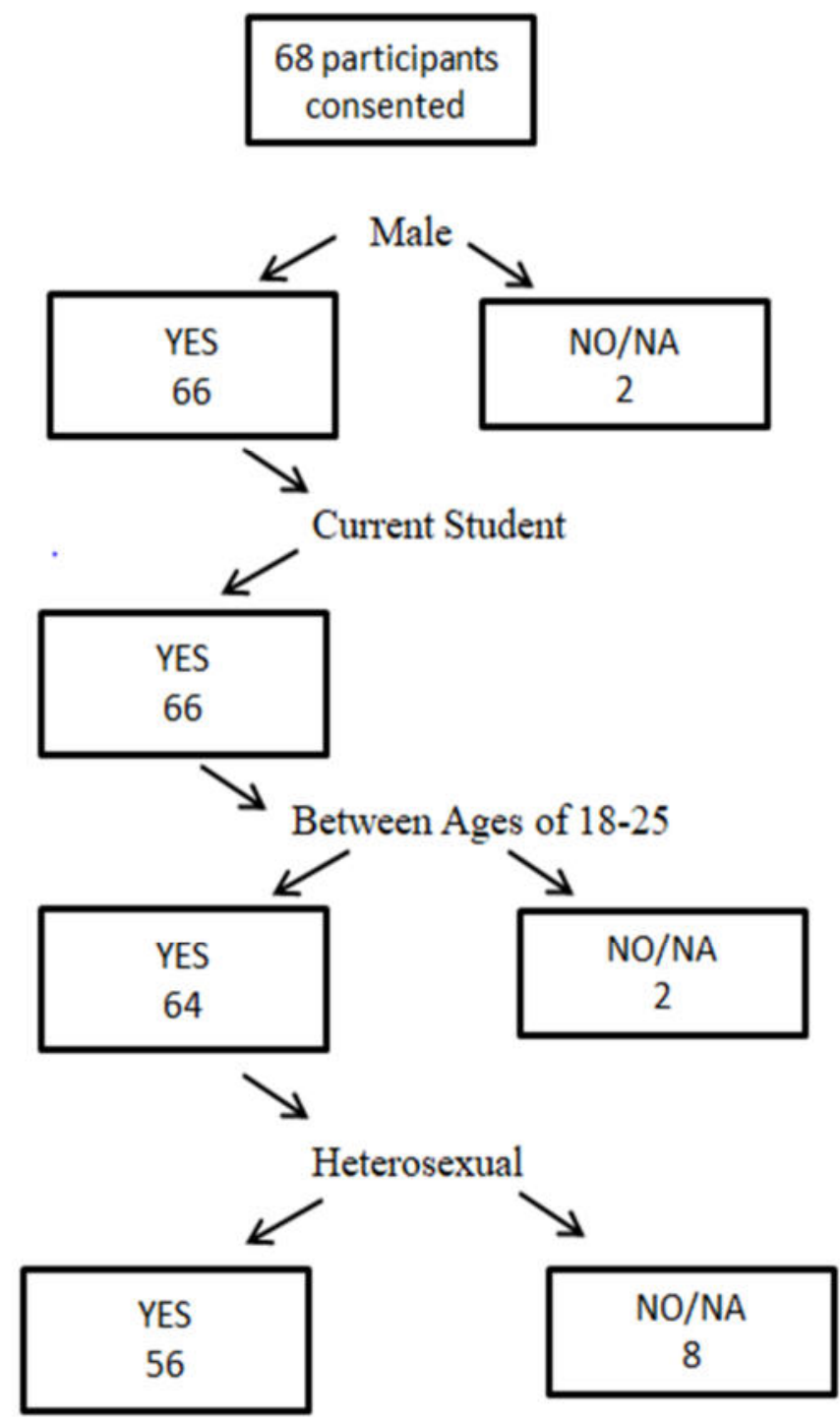

Figure 1: Filter Questions with Participant Answers 
Appendix B

First statement (italics) is original statement from Dr. Willie's work.

Second statement is the rewrite of the current study.

1) One should never tell a girl all his business.

You should never tell a woman all your business.

2) Girls should sometimes share the dating expenses.

Women should sometimes share dating expenses.

3) One should never think that he is the only person dating a girl.

You should never think you are the only man dating the woman you are dating.

4) It does not pay to be nice to a girl.

It does not pay to be nice to a woman

5) If one treats a girl rough she will like him better.

If you treat a woman rough she will like you better.

6) One should never let a girl know that he loves her.

You should never let a woman know that you love her.

7) Men should assume all dating expenses.

You as the man should assume all dating expenses.

8) One should not date a girl if he cannot assume the dating expenses.

You should not date if you cannot pay all of the dating expenses. 
Table 1: Demographics of participants at Albany State University

\begin{tabular}{|l|l|l|}
\hline Age: & $N$ & $\%$ \\
\hline 18 & 10 & \\
\hline 19 & 19 & 39.6 \\
\hline 20 & 3 & \\
\hline 21 & 4 & \\
\hline 22 & 5 & \\
\hline 23 & 4 & \\
\hline 24 & 3 & \\
\hline Class Rank: & & \\
\hline Freshman & 14 & 29.2 \\
\hline Sophomore & 17 & 35.4 \\
\hline Junior & 4 & 8.3 \\
\hline Senior & 3 & 27.1 \\
\hline Relationship Status: & & \\
\hline Not in a relationship & 33 & 68.8 \\
\hline In a relationship & 15 & 31.3 \\
\hline Religious Affiliations: & & \\
\hline Protestant/Christian & 22 & 45.8 \\
\hline Catholic & 3 & 6.3 \\
\hline Non-believer & 9 & 18.8 \\
\hline Other: & & \\
\hline Deist & 1 & 2.1 \\
\hline Non-denominational & 2 & 4.2 \\
\hline Pagan & 1 & 2.1 \\
\hline No Answer & 8 & 16.7 \\
\hline
\end{tabular}

Table 2: Demographics of participants at Morehouse College (1948)

\begin{tabular}{|l|l|l|}
\hline Age: & $N$ & $\%$ \\
\hline $17-18$ & 20 & 18.2 \\
\hline $19-20$ & 36 & 32.7 \\
\hline $21-22$ & 30 & 27.3 \\
\hline $23-24$ & 13 & 11.8 \\
\hline $25-26$ & 8 & 7.3 \\
\hline $27-28$ & 2 & 1.8 \\
\hline $29-30$ & 1 & .9 \\
\hline Class Rank: & & \\
\hline Freshman & 31 & 28.18 \\
\hline Sophomore & 26 & 23.64 \\
\hline Junior & 21 & 19.09 \\
\hline Senior & 32 & 29.09 \\
\hline Religious Affiliations: & & \\
\hline Protestant/Christian & 95 & 86.4 \\
\hline Catholic & 5 & 4.5 \\
\hline Other: & 4 & 3.7 \\
\hline No Answer & 6 & 5.4 \\
\hline Veteran Status: & & \\
\hline Veteran & 39 & 35.5 \\
\hline Non-Veteran & 68 & 61.8 \\
\hline No Answer & 3 & 2.7 \\
\hline
\end{tabular}

Table 2 indicates demographics of Morehouse College participants (Willie, 1949, p. 7-8). 
Table 3: Who taught you about dating relationships with women, \& future marriage?

\begin{tabular}{|l|l|l|}
\hline & $N$ & $\%$ \\
\hline Father/Stepfather & 24 & 50 \\
\hline Mother/Stepmother & 28 & 58.33 \\
\hline Grandparent & 7 & 14.6 \\
\hline Aunt/Uncle & 7 & 14.6 \\
\hline Brother/Sister & 7 & 14.6 \\
\hline Friend & 8 & 16.66 \\
\hline Religious Leader & 3 & 6.25 \\
\hline Godparent & 3 & 6.25 \\
\hline
\end{tabular}

Table 3 lists individual who taught participant about relationships with women, about marriage (Albany State participants).

Table 4: Reasons for dating

\begin{tabular}{|l|l|l|l|l|}
\hline & \multicolumn{2}{|l|}{ ASU } & \multicolumn{2}{l|}{ Morehouse } \\
\hline & $N$ & $\%$ & $N$ & $\%$ \\
\hline Companionship & 35 & 19.1 & 67 & 60.9 \\
\hline To find a suitable mate & 26 & 14.2 & 35 & 31.8 \\
\hline Physical satisfaction & 18 & 9.8 & 35 & 31.8 \\
\hline Natural thing to do & 10 & 5.5 & 17 & 15.4 \\
\hline Keep others from harassing me. & 1 & .5 & 1 & .9 \\
\hline
\end{tabular}

Table 4 lists reasons for dating. 
Table 5: Appropriate Behavior in Courtship

\begin{tabular}{|c|c|c|c|c|}
\hline & Agree & Disagree & Neutral & No Answer \\
\hline \multicolumn{5}{|l|}{$\begin{array}{l}\text { You should never tell a woman all your } \\
\text { business. }\end{array}$} \\
\hline ASU Participants & $n=18,50.0 \%$ & $n=8,22.2 \%$ & $n=10,27.8 \%$ & \\
\hline Morehouse Participants & $n=100,91.0 \%$ & $n=2,1.8 \%$ & $n=5,4.5 \%$ & $n=3,2.7 \%$ \\
\hline \multicolumn{5}{|c|}{$\begin{array}{l}\text { Women should sometimes share dating } \\
\text { expenses. }\end{array}$} \\
\hline ASU Participants & $n=23,63.9 \%$ & $n=5,13.9 \%$ & $n=8,22.2 \%$ & \\
\hline Morehouse Participants & $n=80,72.7 \%$ & $n=10,9.1 \%$ & $n=15,13.6 \%$ & $n=5,4.6 \%$ \\
\hline \multicolumn{5}{|c|}{\begin{tabular}{l|l} 
You should never think you are the only man & \\
dating the woman you are dating. &
\end{tabular}} \\
\hline ASU Participants & $n=18,50 \%$ & $n=8,22.2 \%$ & $n=10,27.8 \%$ & \\
\hline Morehouse Participants & $n=77,70 \%$ & $n=11,10 \%$ & $n=19,17.3 \%$ & $n=3,2.7 \%$ \\
\hline \multicolumn{5}{|l|}{ It does not pay to be nice to a woman. } \\
\hline ASU Participants & $n=12,33.3 \%$ & $n=23,63.9 \%$ & $n=6,16.7 \%$ & \\
\hline Morehouse Participants & $n=8,7.3 \%$ & $n=84,76.4 \%$ & $n=15,13.6 \%$ & $n=3,2.7 \%$ \\
\hline \multicolumn{5}{|l|}{$\begin{array}{l}\text { If you treat a woman rough she will like you } \\
\text { better. }\end{array}$} \\
\hline ASU Participants & $n=7,14.6 \%$ & $n=26,72.2 \%$ & $n=4,11.1 \%$ & \\
\hline Morehouse Participants & $n=10,9.1 \%$ & $n=74,67.3 \%$ & $n=23,20.9 \%$ & $n=3,2.7 \%$ \\
\hline \multicolumn{5}{|l|}{$\begin{array}{l}\text { You should never let a woman know that you } \\
\text { love her. }\end{array}$} \\
\hline ASU Participants & $n=6,12.5 \%$ & $n=26,72.2 \%$ & $n=4,11.1 \%$ & \\
\hline Morehouse Participants & $n=16,14.6 \%$ & $n=54,49.1 \%$ & $n=37,33.6 \%$ & $n=3,2.7 \%$ \\
\hline \multicolumn{5}{|l|}{$\begin{array}{l}\text { You as the man should assume all dating } \\
\text { expenses. }\end{array}$} \\
\hline ASU Participants & $n=12,33.3 \%$ & $n=11,30.6 \%$ & $n=13,36.1 \%$ & \\
\hline Morehouse Participants & $n=30,27.3 \%$ & $n=58,52.7 \%$ & $n=17,15.4 \%$ & $n=5,4.6 \%$ \\
\hline \multicolumn{5}{|l|}{$\begin{array}{l}\text { You should not date if you cannot pay all of } \\
\text { the dating expenses. }\end{array}$} \\
\hline ASU Participants & $n=8,22.2 \%$ & $n=15,41.7 \%$ & $n=13,36.1 \%$ & \\
\hline Morehouse Participants & $n=39,35.4 \%$ & $n=50,45.5 \%$ & $n=18,16.4 \%$ & $n=3,2.7 \%$ \\
\hline
\end{tabular}

